\title{
On exponential fitting of finite difference methods for heat equations
}

\author{
E.O. Tuggen ${ }^{1}$ and C.E. Abhulimen ${ }^{2}$
}

\begin{abstract}
In this article, a new kind of finite difference scheme that is exponentially fitted, inspired from Fourier analysis, for a fourth space derivative was developed for solving diffusion problems. Dispersion relation and local truncation error of the method were discussed. Stability analysis of the method revealed that it is conditionally stable. Compared to the corresponding fourth order classical scheme in the literature, the proposed scheme is efficient and accurate.
\end{abstract}

Mathematics Subject Classification (2020): 65M06, 65N06.

Keywords: Exponential fitting, Finite difference, Local truncation error, Heat equations.

\footnotetext{
1 Department of Mathematics, Ambrose Alli University, Ekpoma, Nigeria.

$2^{2 *}$ Department of Mathematics, Ambrose Alli University, Ekpoma, Nigeria.

*Corresponding author
} 


\section{Introduction}

In this paper, we shall be concerned with the numerical solutions of problems associated with the following reaction-diffusion system.

$$
\frac{\partial u}{\partial t}=M \nabla^{2} u+V(u), \quad u \in \psi \subset \mathfrak{R}^{n}
$$

where $u(x, t)$ is a quantity being diffused in some domain $\Omega \subset \mathfrak{R}^{n}$. In physical applications, $u(x, t), x \in \psi, t>0$ may represent the temperature field, moisture content, vapour concentration etc. $\mathrm{M}$ is a matrix of diffusion coefficients and $\mathrm{V}$ is a vector valued function whose elements are the densities of the substances and represents reaction process. Finally, $\nabla^{2} \equiv \nabla \cdot \nabla$ is the classical n-dimensional Laplace operator [7].

This article is ultimately aimed at developing an exponentially based discrete derivative formula for a fourth derivative, which can be used to model the fourth order diffusion equation in the form:

$\frac{\partial u}{\partial t}=-c \frac{\partial^{4} u}{\partial x^{4}}, \quad x \in[0, L], \quad t \in[0, T]$

with initial condition $: u(x, 0)=u_{\circ}(x)$

and boundary conditions $: u(0, t)=u^{\prime}(0, t)=0$ and $u(L, t)=u^{\prime}(L, t)=0$

Finite difference discretizations of ODEs and PDEs are widely used by researchers due to their easy analysis. Some of their implementation drawbacks include violation of positivity requirements and strict conditions on step sizes which are often encountered in the applications. Few variants of FD scheme have been developed over time. They include the nonstandard finite differences (NSFD) proposed by Mickens [4], whose rules are based on complicated denominators and nonlocal representation of nonlinear terms in the equation [7]. Another alternative is the exponentially fitted models which Paternoster[13] presented in his paper titled, 'an overview of recent literatures in exponential fitting methods'. The similarities and differences between NSFD and EF methods are discussed by Erdoğan[6] and Tadasse[10].

EF method was originally proposed by Liniger and Willoughby [5] as described by Abhulimen[1,2]. The method includes a fitting parameter, the so called frequency that affects the convergence and stability properties of the method. For ordinary differential equations, the problem on how to choose fitting parameters are discussed in $[8,9]$. According to Erdogan [7,11], the full discretization's of partial differential equations has not been well studied.

However, the aim of this paper is to develop an exponentially fitted scheme for (1.2) that can explicitly handle problems in the form of (1.1) numerically for a 
considerable number of time steps. The motivation behind this great attempt is credited to Erdogan [7] and R. J. Leveque [12] who in particular, mentioned the similarity in behavior that exists between (1.1) and (1.2) in his book.

Remark 1.1. It is important to note the second order diffusion equation (1.1) has an important property which the fourth order diffusion equation (1.2) in general does not possess: maximum principle.

\section{Derivatives of the scheme}

We consider the differential equation

$$
y^{\prime}=f(x, y)
$$

Using uniform mesh including $m+1$ points $a=x_{\circ}<x_{1}<\ldots<x_{m}<x_{m+1}=b$ and $h=\frac{b-a}{m+1}$, if $f$ is frozen at $x=x_{i}$ in the subinterval $\left(x_{i-1}, x_{i+1}\right)$ we get an approximation to $(2.1)$ :

$$
\tilde{y}^{\prime}=f_{i}
$$

Adding the terms giving exponential kernel, we obtain

$$
\tilde{y}^{i}-\alpha \tilde{y}=f_{i}-\alpha \tilde{y}_{i}
$$

where $f_{i}=f\left(x_{i}, y_{i}\right), \tilde{y}\left(x_{i}\right)=\tilde{y}_{i}$ and $\alpha \in\left(x_{i-1}, x_{i+1}\right)$. Imposing the continuity conditions $\tilde{y}\left(x_{i}\right)=\tilde{y}_{i}$ and $\tilde{y}\left(x_{i+1}\right)=\tilde{y}_{i+1}$, we obtain:

$$
\frac{\tilde{y}_{i+1}-\tilde{y}_{i}}{\frac{\ell^{\omega h}-1}{\alpha}}=f_{i}
$$

Therefore the new forward difference is

$$
\tilde{y}^{\prime}=\frac{\tilde{y}_{i+1}-\tilde{y}_{i}}{\frac{\ell^{\omega h}-1}{\alpha}}
$$

In the development of the fourth derivative, we consider the differential equation

$$
\tilde{y}^{i v}=f(x, y)
$$

if $f$ is frozen at $x=x_{i}$ in the subinterval $\left(x_{i-2}, x_{i+2}\right)$ we get an approximation to (2.4): $\tilde{y}^{i v}=f_{i}$ 
Adding the terms giving exponential kernel, we obtain

$$
\tilde{y}^{i v}+k^{4} \tilde{y}=f_{i}+k^{4} \tilde{y}_{i}
$$

where $k^{4} \in\left(x_{i-2}, x_{i+2}\right)$. Now, we obtain the four fourth roots associated with the characteristic equation from (2.5) and treating each root as repeated roots, we obtain the general solution in each case as:

$$
\left.\begin{array}{l}
\tilde{y}(x)=c_{1} \ell^{\left(\frac{k+i k}{\sqrt{2}}\right) x}+c_{2} x \ell^{\left(\frac{k+i k}{\sqrt{2}}\right) x}+c_{3} x^{2} \ell^{\left(\frac{k+i k}{\sqrt{2}}\right) x}+c_{4} x^{3} \ell^{\left(\frac{k+i k}{\sqrt{2}}\right) x}+\frac{f_{i}}{k^{4}}+\tilde{y}_{i} \\
\tilde{y}(x)=c_{1} \ell^{\left(\frac{-k+i k}{\sqrt{2}}\right) x}+c_{2} x \ell^{\left(\frac{-k+i k}{\sqrt{2}}\right) x}+c_{3} x^{2} \ell^{\left(\frac{-k+i k}{\sqrt{2}}\right) x}+c_{4} x^{3} \ell^{\left(\frac{-k+i k}{\sqrt{2}}\right) x}+\frac{f_{i}}{k^{4}}+\tilde{y}_{i} \\
\tilde{y}(x)=c_{1} \ell^{\left(\frac{-k-i k}{\sqrt{2}}\right) x}+c_{2} x \ell^{\left(\frac{-k-i k}{\sqrt{2}}\right) x}+c_{3} x^{2} \ell^{\left(\frac{-k-i k}{\sqrt{2}}\right) x}+c_{4} x^{3} \ell^{\left(\frac{-k-i k}{\sqrt{2}}\right) x}+\frac{f_{i}}{k^{4}}+\tilde{y}_{i} \\
\tilde{y}(x)=c_{1} \ell^{\left(\frac{k-i k}{\sqrt{2}}\right) x}+c_{2} x \ell^{\left(\frac{k-i k}{\sqrt{2}}\right) x}+c_{3} x^{2} \ell^{\left(\frac{k-i k}{\sqrt{2}}\right) x}+c_{4} x^{3} \ell^{\left(\frac{k-i k}{\sqrt{2}}\right) x}+\frac{f_{i}}{k^{4}}+\tilde{y}_{i}
\end{array}\right\}
$$

To obtain the exponentially fitted for (2.4), we shall impose some continuity conditions on each of equations in (2.6):

$\tilde{y}\left(x_{i-2}\right)=\tilde{y}_{i-2}, \tilde{y}\left(x_{i-1}\right)=\tilde{y}_{i-1}, \tilde{y}\left(x_{i}\right)=\tilde{y}_{i}, \tilde{y}\left(x_{i+1}\right)=\tilde{y}_{i+1}$ and $\tilde{y}\left(x_{i+2}\right)=\tilde{y}_{i+2}$, which will always lead to five (5) algebraic equations for each case. After a rigorous algebraic manipulations and simplifications, we obtain the required exponentially fitted fourth derivative quotient given by:

$$
\tilde{y}^{i v}\left(x_{i}\right)=f_{i}=\frac{\tilde{y}_{i+2}-4 \tilde{y}_{i+1}+6 \tilde{y}_{i}-4 \tilde{y}_{i-1}+\tilde{y}_{i-2}}{\frac{8 \cos \left(\frac{\sqrt{2}}{2} k h\right) \cosh \left(\frac{\sqrt{2}}{2} k h\right)-2 \cos (\sqrt{2} k h) \cosh (\sqrt{2} k h)-6}{k^{4}}}
$$

\section{Fourth Order Diffusion}

$$
\begin{gathered}
\text { We shall conside } \\
\frac{\partial u}{\partial t}=-c \frac{\partial^{4} u}{\partial x^{4}}, \quad-\pi<x<\pi
\end{gathered}
$$

with initial condition $u(x, 0)=u_{\mathrm{o}}(x)$ and periodic boundary conditions. We shall assume a trial solution of the form $u(x, t)=X(x) T(t)$, where $X(x)$ is a function of $x$ only and $T(t)$ is a function of $t$ only. 
The separation of variables method gives

$$
\frac{T^{\prime}(t)}{c T(t)}=\frac{-X^{i v}(x)}{X(x)}=-k^{4}
$$

Simplifying the above, we obtain the time and spatial components of the solution as $T=\ell^{-c k^{4} t}=\ell^{\alpha t}\left(\right.$ where $\left.\alpha=-c k^{4}\right)$ and $(A \sin k x+B \cos k x+C \sinh k x+D \cosh k x)$ respectively. The relation between time and spatial components is called dispersion relation [7] and in this case it is given by

$$
\alpha=-c k^{4}
$$

The relation (3.1) is never taken into account explicitly in the classical finite difference methods as described by Erdoğan [7]. Our contribution here, is to consider (3.1) in the construction of finite difference schemes. We recall that exponentially fitted methods and nonstandard finite differences avail us with a useful tool in the form of denominators in terms of non-polynomial functions $[7,4]$. Therefore we present forward time derivative and fourth space derivative for (1.2) to obtain an explicit exponentially fitted scheme given by:

$$
\frac{\frac{u_{i}^{j+1}-u_{i}^{j}}{\ell^{\alpha_{i}^{j} \Delta t}-1}}{\alpha_{i}^{j}}=-c \cdot \frac{u_{i+2}^{j}-4 u_{i+1}^{j}+6 u_{i}^{j}-4 u_{i-1}^{j}+u_{i-2}^{j}}{\frac{8 \cos \left(\frac{\sqrt{2}}{2} k_{i}^{j} \Delta x\right) \cosh \left(\frac{\sqrt{2}}{2} k_{i}^{j} \Delta x\right)-2 \cos \left(\sqrt{2} k_{i}^{j} \Delta x\right) \cosh \left(\sqrt{2} k_{i}^{j} \Delta x\right)-6}{k_{i}^{j^{4}}}}
$$

where $\alpha$ and $k$ are evaluated at the $(i, j)^{\text {th }}$ mesh point.

The assignment of $\alpha$ and $k$ and the relation between these parameters are of paramount importance. In this paper, we shall describe a parameter selection strategy that is based on dispersion relation and local error formula in consonance with the work of Erdogan [7].

The local error of (3.2) can be written as:

$$
T_{i}^{j}=\frac{1}{2} \Delta t\left(\left(u_{t t}\right)_{i}^{j}-\omega_{i}^{j} \cdot\left(u_{t}\right)_{i}^{j}\right)+\frac{c}{6} \Delta x^{4}\left(\left(u_{x x x x x}\right)_{i}^{j}+k_{i}^{j^{4}} \cdot\left(u_{x x x x}\right)_{i}^{j}\right)+O\left(\Delta t^{2}, \Delta x^{2}\right)
$$

Where the derivatives $\left(u_{t}\right)_{i}^{j},\left(u_{x}\right)_{i}^{j}$ and other higher derivatives are computed at the $\operatorname{point}\left(x_{i}, t_{j}\right)$. 
The selections

$$
k_{i}^{j^{4}}=-\frac{\left(u_{x x x x x x x x}\right)_{i}^{j}}{\left(u_{x x x x}\right)_{i}^{j}} \quad \text { and } \quad \omega_{i}^{j}=\frac{\left(u_{t t}\right)_{i}^{j}}{\left(u_{t}\right)_{i}^{j}}
$$

That makes the first two terms zero in the local error formula is of importance here. This is because the selections give the dispersion relation () of the fourth order diffusion by considering

$$
\omega_{i}^{j}=\frac{\left(u_{t t}\right)_{i}^{j}}{\left(u_{t}\right)_{i}^{j}}=\frac{c^{2}\left(u_{x x x x x x x x}\right)_{i}^{j}}{-c\left(u_{x x x x}\right)_{i}^{j}}=-c k_{i}^{j^{4}}
$$

In the implementation of (3.5), the classical finite difference approximation of $u_{x x x x x x x}$ and $u_{x x x x}$ might be employed as follows for computing $k$ numerically:

$k_{i}^{j^{4}}=\frac{1}{576 \Delta x^{4}} \cdot \frac{\left(\begin{array}{l}u_{i+8}^{j}-84 u_{i+4}^{j}+1344 u_{i+2}^{j}-4096 u_{i+1}^{j}+5670 u_{i}^{j} \\ -4096 u_{i-1}^{j}+1344 u_{i-2}^{j}-84 u_{i-4}^{j}+u_{i-8}^{j}\end{array}\right)}{\left(u_{i+2}^{j}-4 u_{i+1}^{j}+6 u_{i}^{j}-4 u_{i-1}^{j}+u_{i-2}^{j}\right)}, \quad \alpha_{i}^{j}=-c k_{i}^{j^{4}}$

However, in this paper rather than applying the frequency search algorithm in the implementation, we shall restrict our ambition by making use of settings for picking suitable values for $k$ and $\alpha$.

\subsection{Stability Analysis}

Definition 3.1. Stability is the property which a numerical method possesses that keeps its errors bounded as the computation advances [3].

Now, to establish the stability of our method, we must understand that both classical explicit finite difference scheme and our exponentially fitted scheme for the fourth order diffusion equation can be written as:

$u_{i}^{j+1}=u_{i}^{j}-\lambda\left(u_{i+2}^{j}-4 u_{i+1}^{j}+6 u_{i}^{j}-4 u_{i-1}^{j}+u_{i-2}^{j}\right)$

Where;

$$
\lambda_{F D}=\lambda=\frac{c \Delta t}{\Delta x^{4}}
$$

and

$$
\lambda=\lambda_{E F M}=c \cdot \frac{\frac{\ell^{\omega_{i}^{j} \Delta t}-1}{\omega_{i}^{j}}}{\frac{8 \cos \left(\frac{\sqrt{2}}{2} k_{i}^{j} \Delta x\right) \cosh \left(\frac{\sqrt{2}}{2} k_{i}^{j} \Delta x\right)-2 \cos \left(\sqrt{2} k_{i}^{j} \Delta x\right) \cosh \left(\sqrt{2} k_{i}^{j} \Delta x\right)-6}{k_{i}^{j^{4}}}}
$$


Stand for the Courant-Frederichs-Lewy (CFL) numbers and $\lambda$ for classical finite difference and exponentially fitted schemes. After the dispersion relation $\alpha_{i}^{j}=-c k_{i}^{j^{4}}$ is substituted into $\lambda_{E F M}$, we find that the limit

$$
\lim _{k_{i}^{j} \rightarrow 0} \lambda_{E F M}=\frac{c \Delta t}{\Delta x^{4}}=\lambda_{F D}
$$

Hence just like the case for the second order diffusion [7], this tells us that the classical finite difference formulation is employed in the implementation in case very small $k$ be noticed.

Now, we obtain the stability of our method by using

$$
u_{i}^{j+1}=u_{i}^{j}-\lambda_{E F M}\left(u_{i+2}^{j}-4 u_{i+1}^{j}+6 u_{i}^{j}-4 u_{i-1}^{j}+u_{i-2}^{j}\right)
$$

And assume that our numerical scheme admits solutions of the form:

$$
Z_{m}^{n}=E^{n}(k) \ell^{i m k h}
$$

Where $k$ is the wave number and $i=\sqrt{-1}$ and define

$$
G(k)=\frac{E^{n+1}(k)}{E^{n}(k)}
$$

as amplification factor which governs the growth of the Fourier component $E(k)$. The Von Neumann [15], stability condition requires that the modulus of (3.10) must be less than or equal to unity. After a cumbersome algebraic manipulations, it is easy to verify that this requirement is satisfied when

$$
\lambda_{E F M} \leq \frac{1}{2(\cos k h-1)^{2}} \quad, \quad \text { provided } k h \neq 0
$$

From above, we can say that the new exponentially fitted method is conditionally stable and the method converges to the solution of (1.2) with rate of converges of $\left(\Delta t+\Delta x^{4}\right)$, provided that

$$
\frac{c \Delta t}{\Delta x^{4}} \leq \frac{1}{2(\cos k h-1)^{2}}
$$

It should be noted that the stability of the method depends not only on the step sizes but also on the parameters of the method. 


\subsection{Matrix representation of the method}

The proposed method is explicitly represented in the following matrix form for the purpose of application [12]. We first present the initial and boundary conditions associated with the method:

$$
\left.\begin{array}{c}
u_{i}^{0}=u_{0}\left(x_{i}\right) \quad ; i=0,1,2, \ldots, m, m+1 \\
u_{0}^{j}=u_{-1}^{j}+u_{1}^{j}=u_{-2}^{j}+u_{2}^{j}=0 \\
u_{m+1}^{j}=u_{m}^{j}+u_{m+2}^{j}=u_{m-1}^{j}+u_{m+3}^{j}=0 \\
j=0,1,2, \ldots, n+1
\end{array}\right\}
$$

Hence, we have

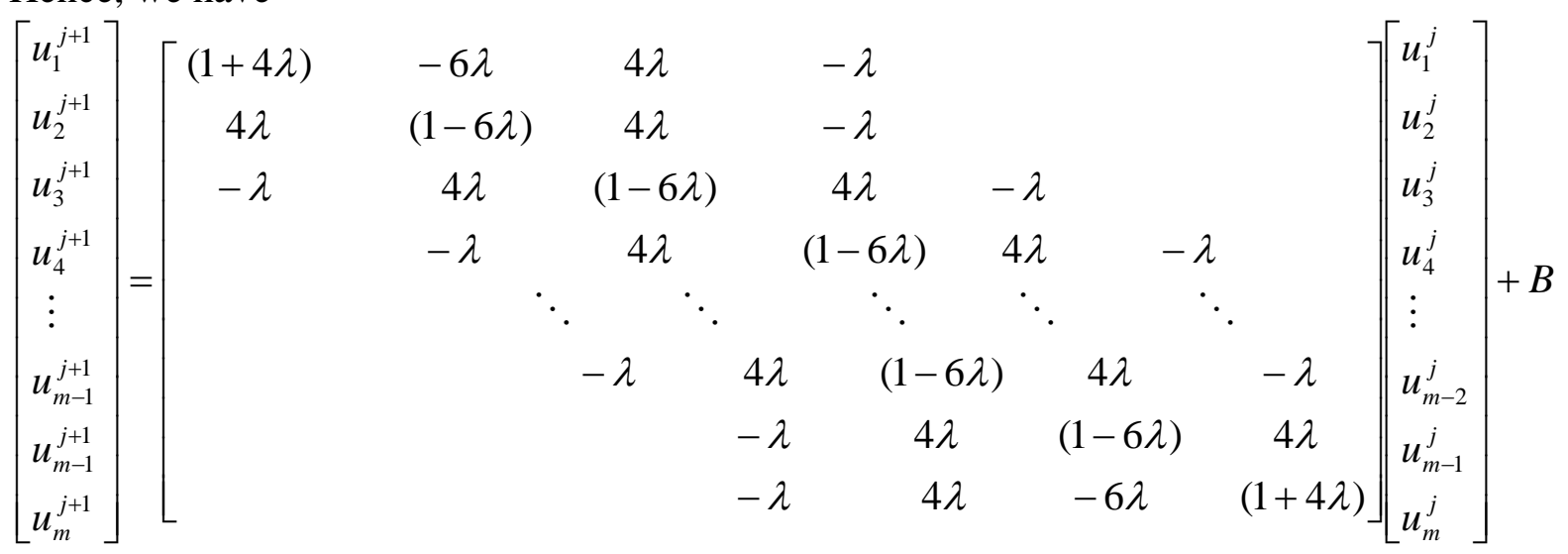

Where B is the column matrix containing boundary values and is given by

$$
B=\left[\begin{array}{lllllll}
-\lambda\left(u_{-1}^{j}+u_{1}^{j}\right) & -\lambda\left(u_{-2}^{j}+u_{2}^{j}\right) & 0 & \ldots & 0 & -\lambda\left(u_{m-1}^{j}+u_{m+3}^{j}\right) & -\lambda\left(u_{m}^{j}+u_{m+2}^{j}\right)
\end{array}\right]^{T}
$$

which can be written as

$$
F=A U+B
$$

The nearly pentadiagonal matrix on the right hand side of (5.2) is known (and constant) once the time-step, $\Delta t$ is chosen. The entries in the column matrix B are expected to be all zeros in this case. The known values of $u$ at $u_{i}^{j}=f\left(x_{i}\right), i=1, \ldots, m$, are multiplied by this nearly pentadiagonal matrix to obtain new values of $u$ at $u_{i}^{j+1}$. 


\section{Numerical examples}

\section{Problem 1}

We consider the heat equation from Richard L. Burden and J. Douglas Faires [14].

$$
\frac{\partial u}{\partial t}-\frac{1}{\pi^{2}} \frac{\partial^{2} u}{\partial x^{2}}=0, \quad 0<x<1,0<t
$$

with boundary conditions

$$
u(0, t)=u(1, t)=0, \quad 0<t,
$$

and initial condition

$$
u(x, 0)=\cos \pi\left(x-\frac{1}{2}\right), \quad 0 \leq x \leq 1 .
$$

The initial condition leads to the exact solution $u(x, t)=\ell^{-t} \cos \pi\left(x-\frac{1}{2}\right)$.

We will use $\Delta x=0.1$ and $\Delta t=0.00004$, and compare our results at $t=0.0004$ to the exact solution. In Table 6.1, the EF method with settings $k=1$ and $\omega=-10^{6}$ gives an exact solution up to machine accuracy. We denote classical method and

\begin{tabular}{|c|c|c|c|c|c|}
\hline$x_{i}$ & $\begin{array}{c}\text { Exact } \\
\text { Solution (ES) } \\
u\left(x_{i}, 0.0004\right)\end{array}$ & CM 4 & $\mathrm{AE} 4$ & $|E S-C M 4|$ & $|E S-A E 4|$ \\
\hline 0 & 0 & 0 & 0 & 0 & 0 \\
\hline 0.1 & 0.30889338 & 0.30610277 & 0.30895909 & $2.791 \times 10^{-3}$ & $6.571 \times 10^{-5}$ \\
\hline 0.2 & 0.58755017 & 0.58487103 & 0.58772743 & $2.679 \times 10^{-3}$ & $1.773 \times 10^{-4}$ \\
\hline 0.3 & 0.80869345 & 0.80586006 & 0.80893824 & $2.833 \times 10^{-3}$ & $2.448 \times 10^{-4}$ \\
\hline 0.4 & 0.95067616 & 0.94742368 & 0.95096375 & $3.252 \times 10^{-3}$ & $2.876 \times 10^{-4}$ \\
\hline 0.5 & 0.99960008 & 0.99615138 & 0.99990207 & $3.449 \times 10^{-3}$ & $3.020 \times 10^{-4}$ \\
\hline 0.6 & 0.95067616 & 0.94742368 & 0.95096377 & $3.252 \times 10^{-3}$ & $2.876 \times 10^{-4}$ \\
\hline 0.7 & 0.80869345 & 0.80586006 & 0.80893822 & $2.833 \times 10^{-3}$ & $2.448 \times 10^{-4}$ \\
\hline 0.8 & 0.58755017 & 0.58487103 & 0.58772743 & $2.679 \times 10^{-3}$ & $1.773 \times 10^{-4}$ \\
\hline 0.9 & 0.30889338 & 0.30610278 & 0.30895909 & $2.791 \times 10^{-3}$ & $6.571 \times 10^{-5}$ \\
\hline 1.0 & 0 & 0 & 0 & 0 & 0 \\
\hline
\end{tabular}
present method respectively by $\mathrm{CM} 4$ and AE4 in our numerical results.

Table 4.1: Table of solution values at $\mathrm{t}=\mathbf{0 . 0 0 0 4}$. 
From the numerical results of Problem 1 presented in Table (4.1), we observed that for various step sizes our proposed scheme competes favorably with the existing classical method which is an existing method in the literature.

\section{Problem 2}

We consider the diffusion equation from Richard L. Burden and J. Douglas Faires [14].

$$
\frac{\partial u}{\partial t}-\frac{4}{\pi^{2}} \frac{\partial^{2} u}{\partial x^{2}}=0, \quad 0<x<4,0<t
$$

with boundary conditions

$$
u(0, t)=u(4, t)=0, \quad 0<t,
$$

and initial condition

$$
u(x, 0)=\sin \frac{\pi}{4} x\left(1+2 \cos \frac{\pi}{4} x\right), \quad 0 \leq x \leq 4 .
$$

The initial condition leads to the exact solution $u(x, t)=\ell^{-t} \sin \frac{\pi}{2} x+\ell^{-\frac{t}{4}} \sin \frac{\pi}{4} x$.

We will use $\Delta x=0.2$ and $\Delta t=0.0004$, and compare our results at $t=0.0012$ to the exact solution. In Table 5.3, the EF method with settings $k=1$ and $\omega=-10^{4}$ gives an exact solution up to machine accuracy. We denote classical method and present method respectively by $\mathrm{CM} 4$ and $\mathrm{AE} 4$ in our numerical results. 
Table 4.2: Table of solution values at $\mathbf{t}=\mathbf{0 . 0 0 1 2}$.

\begin{tabular}{|c|c|l|l|l|l|}
\hline$x_{i}$ & $\begin{array}{c}\text { Exact Solution (ES) } \\
u\left(x_{i}, 0.0012\right)\end{array}$ & $\mathrm{CM} 4$ & $\mathrm{AE} 4$ & $|E S-C M 4|$ & $|E S-A E 4|$ \\
\hline 0 & 0 & 0 & 0 & 0 & 0 \\
\hline 0.2 & 0.46503395 & 0.46337838 & 0.46499753 & $1.656 \times 10^{-3}$ & $3.642 \times 10^{-5}$ \\
\hline 0.4 & 0.89600465 & 0.89472917 & 0.89634827 & $1.275 \times 10^{-3}$ & $3.436 \times 10^{-4}$ \\
\hline 0.6 & 1.2619011 & 1.2606032 & 1.2624133 & $1.298 \times 10^{-3}$ & $5.122 \times 10^{-4}$ \\
\hline 0.8 & 1.5375249 & 1.5359892 & 1.5381360 & $1.536 \times 10^{-3}$ & $6.111 \times 10^{-4}$ \\
\hline 1.0 & 1.7056954 & 1.7040638 & 1.7063602 & $1.632 \times 10^{-3}$ & $6.648 \times 10^{-4}$ \\
\hline 1.2 & 1.7586903 & 1.7571570 & 1.7593575 & $1.533 \times 10^{-3}$ & $6.672 \times 10^{-4}$ \\
\hline 1.4 & 1.6987860 & 1.6975054 & 1.6994052 & $1.281 \times 10^{-3}$ & $6.192 \times 10^{-4}$ \\
\hline 1.6 & 1.5378515 & 1.5369562 & 1.5383787 & $8.953 \times 10^{-4}$ & $5.272 \times 10^{-4}$ \\
\hline 1.8 & 1.2960384 & 1.2956242 & 1.2964399 & $4.142 \times 10^{-4}$ & $4.015 \times 10^{-4}$ \\
\hline 2.0 & 0.99970004 & 0.99981569 & 0.99995475 & $1.157 \times 10^{-4}$ & $2.547 \times 10^{-4}$ \\
\hline 2.2 & 0.67874559 & 0.67938846 & 0.67884740 & $6.429 \times 10^{-4}$ & $1.018 \times 10^{-4}$ \\
\hline 2.4 & 0.36369089 & 0.36480622 & 0.36364819 & $1.115 \times 10^{-3}$ & $4.270 \times 10^{-5}$ \\
\hline 2.6 & 0.08269244 & 0.084179256 & 0.082527206 & $1.487 \times 10^{-3}$ & $1.652 \times 10^{-4}$ \\
\hline 2.8 & -0.14114166 & -0.13942139 & -0.14139662 & $1.720 \times 10^{-3}$ & $2.550 \times 10^{-4}$ \\
\hline 3.0 & -0.29190605 & -0.29011118 & -0.29221063 & $1.795 \times 10^{-3}$ & $3.046 \times 10^{-4}$ \\
\hline 3.2 & -0.36230704 & -0.36063375 & -0.36261862 & $1.673 \times 10^{-3}$ & $3.116 \times 10^{-4}$ \\
\hline 3.4 & -0.35419242 & -0.35278725 & -0.35447301 & $1.405 \times 10^{-3}$ & $2.806 \times 10^{-4}$ \\
\hline 3.6 & -0.27815594 & -0.27682947 & -0.27834355 & $1.326 \times 10^{-3}$ & $1.876 \times 10^{-4}$ \\
\hline 3.8 & -0.15225887 & -0.15064370 & -0.15215777 & $1.615 \times 10^{-3}$ & $1.011 \times 10^{-4}$ \\
\hline 4.0 & 0 & 0 & 0 & 0 & 0 \\
\hline
\end{tabular}

From the numerical results of Problem 2 presented in Table (4.2), we observed that for various step sizes, our proposed scheme competes favorably with the classical method which is an existing method in the literature.

\section{Conclusion}

Following the numerical results presented so far in this article, simply show that our newly derived EF method that is first order in time and fourth order in space which has a second order accuracy dominance and competes favorably with the classical method of the fourth order diffusion equation with same order of accuracy and do behave much like the second order heat equation.

In conclusion, our new EF method can adequately solve heat problems for a considerable number of initial time steps. Though, time stepping is generally the only major challenge for the explicit method of solving heat problems using the fourth order diffusion equation.

Acknowledgements: I would like to thank Professor Cletus E. Abhulimen for his illuminating assistance, work ethics and useful guidance. 


\section{References}

[1] Abhulimen, C.E. and Omeike, G.E. (2011). A sixth-order exponentially fitted scheme for the numerical solution of systems of ordinary differential equations, Journal of Applied Mathematics \& Bioinformatics, vol., no. 1, 175-186.

[2] Abhulimen, C.E. and Okunuga, S.A. (2008). Exponentially fitted second derivative multiple method for stiff initial value problem for ODEs, Journal of Engineering Science and Applications, 5, 36-49.

[3] Abhulimen, C.E (2006). On exponentially fitted multi-derivative numerical methods for stiff initial values problems in ODEs. (Ph.D. Thesis), Dept. of Mathematics, University of Benin, Nigeria.

[4] Mickens, R.E. (1994). Nonstandard finite difference models of differential equations, World Scientific, Singapore.

[5] Liniger, W. and Willoughby R.A. (1970). Efficient integration methods for stiff systems of ordinary differential equations, SIAM J. Numer Annal,7, 4765.

[6] Erdoğan U. and Özis T. (2011). A smart nonstandard finite difference scheme for second order nonlinear boundary value problems, Journal of Computational Physics, 230, 6464-6474.

[7] Erdoğan,U., Akarbulut, K. and Tan, N.O. (2016). Exponentially fitted finite difference schemes for reaction- diffusion equations, Mathematical and Computational Applications, 21-32.

[8] Erdoğan, U. and Kocak, H. (2013). Numerical study of the asymptotics of the second Painlevé by a functional fitting method, Math. Methods Appl. Sci., 36, 2347-2352.

[9] Ramos, H. and Vigo-Aguiar, J. (2010). On the frequency choice in trigonometrically fitted methods, Appl. Math. Lett., 23, 1378-1381.

[10] Tadesse, G. and Kalyani, P. (2016). Numerical Solution of Convection Diffusion Problem using nonstandard finite difference methods and comparison with standard finite difference methods, IOSR Journal of Mathematics (IOSR-JM), vol. 12, 94-109.

[11] Causon, D.M. and Mingham, C.G. (2010). Introductory finite difference methods for PDEs, Ventus Publishing ApS.

[12] Leveque, R. J. (2007). Finite difference methods for ordinary and partial differential equations, Society for industrial and applied mathematics, University science center, Philadelphia.

[13] Paternoster, B. (2012). Present state-of-the-art in exponential fitting: A contribution dedicated to Liviu Ixaru on his 70th birthday, Comput. Phys. Commun., 183, 2499-2512.

[14] Burden, R.L. and Faires, J.D. ( 2011). Numerical Analysis, Brooks/Cole, Cengage Learning.

[15] Smith, G.D. (1985). Numerical solutions of partial differential equations: finite difference methods, Clarendon Press, Oxford. 